\begin{tabular}{|c|c|c|}
\hline$\underset{P U B L I C A T I O N}{\operatorname{NIF}}$ & $\begin{array}{l}\text { International Journal of Advanced Engineering, Management and } \\
\text { Science (IJAEMS) } \\
\text { Peer-Reviewed Journal } \\
\text { ISSN: } 2454-1311 \text { / Vol-7, Issue-10; Oct, } 2021 \\
\text { Journal Home Page: https://ijaems.com/ } \\
\text { Article DOI: } \text { https://dx.doi.org/10.22161/ijaems.710.4 }\end{array}$ & (1) \\
\hline
\end{tabular}

\title{
Hedgers Competition in Financial Market
}

\author{
Letian Jiao ${ }^{1}$,Tianyu Zhang ${ }^{2}$,Haitao Chen $^{3}$
}

\begin{abstract}
${ }^{1}$ China Economics and Management Academy, Central University of Finance and Economics, Beijing, China
${ }^{2}$ China Economics and Management Academy, Central University of Finance and Economics, Beijing, China

${ }^{3}$ School of Economics, Central University of Finance and Economics, Beijing, China

Correspondence: Haitao Chen, School of Economics Central University of Finance and Economics, Beijing, China.
\end{abstract}

Received: 11 Sep 2021; Received in revised form: 15 Oct 2021; Accepted: 21 Oct 2021; Available online: 31 Oct 2021

\begin{abstract}
We analyze a two-period model with two kinds of hedgers who have different kinds of non-tradable risky asset to hedge. For holding the asset whose payoff is related to a tradable risky asset, they can derive some different private information about this tradable risky asset. Both hedgers have demand to buy risky asset for the purpose of speculating and hedging. In date 1, they get the information about their non-tradable asset position, private signal of tradable risky asset' payoff and decide how much tradable risky asset they want to hold. They can estimate each other's private information through equilibrium price. We also measure the information passing effect of price.
\end{abstract}

Keywords-Hedgers Competition, Private Information, Equilibrium Price.

\section{INTRODUCTION}

In financial market, we can always see different investors trade in a kind of tradable risky asset to hedging the risk exposure which brought by holding the illiquid asset. For instance, in future market, different participants have different hedging demand. They use future to hedge the loss that price volatility of their position leads. Such as farmers enter the crops future market and short it to avoid the loss that crops price decreases. Meanwhile, big restaurants and food manufacturers enter the crops future market and long it to avoid the loss that crops price increases. They may also have speculation demand to take more risk through future market if they can accurately estimate the changing of future price. Because of holding different illiquid asset, they can gain some private information that counterparts do not know. To illustrate, farmers can better understand the weather, fertilize, and some other factors which can influence the supply of crops and influence the future price further. As for those who long crops future, they may have more insight into the demand of crops which influence crops future price. Thus, the hedgers may have different private information according to the payoff of tradable risky asset. Hedgers do not know each other's private information about risky asset's payoff, they also do not know how many non-tradable assets their counterparts need to hedge. Through market clearing, they can gain some information about counterparts' private signal and their personal holding from equilibrium price. The relativity between tradable risky asset and non-tradable asset can either be positive and negative. We not only analyze hedgers' hedging demands, we also analyze their speculation demands. And we study the price informativeness and what factors influence the information learning accuracy.

In our paper, we analyze a two-period model with two kinds of hedgers who have different kinds of non-tradable risky asset to hedge. For holding the asset whose payoff is related to a tradable risky asset, they can derive some 
different private information about this tradable risky asset. Both hedgers have demand to buy risky asset for the purpose of speculating and hedging. In date 1 , they get the information about their non-tradable asset position, private signal of tradable risky asset' payoff and decide how much tradable risky asset they want to hold. They can estimate each other's private information through equilibrium price. We also study what factor influence the information passing effect of price and risk premium.

This paper is organized as follow. In section 2, literature review is given. In section 3 , we introduce the model setting and assumption. In section 4, we define the market equilibrium and analyze how hedgers learn from market price and make their decision. In section 5, we study how equilibrium price is determined and the factors that influence the price. In section 6 and 7, we consider price informativeness and risk premium respectively. In section 8 , we give our conclusion.

\section{LITERATURE REVIEW}

Liu and Wang (2019) use a random holding non-tradable asset position and analyze a model with hedgers, speculators and market maker. The hedgers are endowed with a random position on a non-tradable risky asset which is corelated with a tradable asset. They have both hedging demand speculation demand and speculators only have speculation demand. Liu and Wang (2016) analyze the market trading under asymmetric information incurred by holding a kind of non-tradable asset. Goldstein, Li and Yang (2014) study a model that different traders have different investment opportunity. That leads to different trading purpose in same financial market, some traders trade for speculation and others trade for hedging. We use same measure to analyze price informativeness and risk premium as Goldstein, Li and Yang. Easley, O'Hara and Yang (2014) study the ambiguity effect of hedging fund investment strategies on asset price and aggregate welfare by letting correlation between asset be unknown to opaque traders. Grossman and Stiglitz (1980) give a fundamental framework of the CARA-normal REE model. Admati (1985) study multiple risk asset and their interaction and analyze a noisy expectation equilibrium. Huand Qin (2013) analyze welfare effect of information acquisition in a competitive financial market with diverse information and rational expectation. Bessembinder (1992) studies the relation between agricultural future return and hedgers' holding.Biais, Bossaerts and Spatt (2010) study the implications of information asymmetric for equilibrium asset pricing and portfolio choice both theoretically and empirically. Easley and O'hara (2004) analyze the influence of information on cost of capital.

We introduce a model that both participants have hedging demand, and there exists asymmetric information but no one have an information advantage. Investors use their own private information and equilibrium price to estimate other information in competitive market.

\section{MODEL}

We analyze a two discreet periods (date 1 and date 2) economy and a continuum of hedgers indexed by the unit interval $[0,1]$. There exist two kinds of hedgers labeled type 1 and type 2, which are endowed with different kind of non-tradable risky asset 1 and asset 2 respectively. The fraction of type 1 hedgers is $\mu$ and thus the fraction of type 2 hedgers is $1-\mu$.They all have CARA utility function with risk aversion coefficient $\gamma$.There is only one kind of tradable risky asset (We call it asset $m$ ) or may be optimal market portfolio in financial market.

\subsection{Asset}

\subsubsection{Tradable Asset}

Risky asset $m$ is the only tradable asset in the market. And its payoff $\tilde{v}$ is constituted by five parts: prior belief mean payoff of asset $\mathrm{m} \bar{v}$, private information for type 1 hedgers $\tilde{\theta}_{1}$, private information for type 2 hedgers $\tilde{\theta}_{2}$, disruption corelated with asset $1 \quad \tilde{\varepsilon}_{1}$, disruption corelated with asset 2 $\tilde{\varepsilon}_{2}$, that is

$$
\tilde{v}=\bar{v}+\tilde{\theta}_{1}+\tilde{\theta}_{2}+\tilde{\varepsilon}_{1}+\tilde{\varepsilon}_{2}
$$

where $\bar{v}>0$, $\tilde{\theta}_{1} \sim N\left(0, \sigma_{\theta 1}^{2}\right), \tilde{\theta}_{2} \sim N\left(0, \sigma_{\theta 2}^{2}\right), \tilde{\varepsilon}_{1} \sim N\left(0, \sigma_{\varepsilon 1}^{2}\right), \tilde{\varepsilon}_{2} \sim N\left(0, \sigma_{\varepsilon 2}^{2}\right)$. And they are all independent.

Let precision of $\tilde{\theta}_{1}$ be defined as $\tau_{\theta 1}=\frac{1}{\sigma_{\theta 1}^{2}}$, precision of $\tilde{\theta}_{2}$ be $\tau_{\theta 2}=\frac{1}{\sigma_{\theta 2}^{2}}$, precision of $\tilde{\varepsilon}_{1}, \tilde{\varepsilon}_{2}$ be $\tau_{\varepsilon 1}=\frac{1}{\sigma_{\varepsilon 1}^{2}}$ and $\tau_{\varepsilon 2}=\frac{1}{\sigma_{\varepsilon 2}^{2}}$ respectively. We let supply of tradable asset be $n$ unit. The price of asset mpis determined by demand and supply endogenously. 


\subsubsection{Non-tradable Asset}

Asset 1 and asset 2 are non-tradable in the financial market, they can only be held to maturity at date 2 and realize their payoff. The payoff of asset 1 is $\tilde{u}_{1}$ that follows $N\left(0, \sigma_{u 1}^{2}\right)$. The payoff of asset 2 is $\tilde{u}_{2}$ that follows $N\left(0, \sigma_{u 2}^{2}\right)$.

The correlation between asset 1 and asset mis $\rho_{1}$, the correlation between asset 2 and asset $m$ is $\rho_{2}$. Both $\rho_{1}$ and $\rho_{2}$ belongs to $[-1,1]$.

\subsubsection{Free-risk asset}

The free-risk asset generates no interest.

\subsection{Hedgers}

Type 1 hedgers are endowed with $\tilde{X}_{1}$ units of asset 1 at date 1 , type 2 hedgers are endowed with $\tilde{X}_{2}$ units of asset 2. $\tilde{X}_{1}$ and $\tilde{X}_{2}$ follow normal distribution $N\left(0, \sigma_{X 1}^{2}\right)$ and $N\left(0, \sigma_{X 1}^{2}\right)$ respectively. And $\tilde{X}_{1}$ and $\tilde{X}_{2}$ are all independent.

Let precision of $\tilde{X}_{1}$ be $\tau_{X 1}=\frac{1}{\sigma_{X 1}^{2}}$ and precision of $\tilde{X}_{2}$ be $\tau_{X 2}=\frac{1}{\sigma_{X 2}^{2}}$. Both types of hedgers born with currency wealth $W$ that can be used to buy asset $m$. And both types of hedgers have utility function $u=-e^{-\gamma W_{i}^{2}}$, where $W_{i}^{t}$ denote the wealth type $i$ hedgers have at date $t$. Thus, we have

$=d_{f i}+d_{i} p$

$=d_{i} \tilde{v}+d_{f i}+\tilde{X}_{i} \tilde{u}_{i}$

Combine (1) and (2), we have

$$
W_{i}^{2}=d_{i}(\tilde{v}-p)+W
$$

$$
+\tilde{X}_{i} \tilde{u}_{i}
$$

$i \in\{1,2\}$.

\subsection{Information structure}

\subsubsection{Date 1}

Type 1 hedger get a private signal $\tilde{\theta}_{1}$ and their position on non-tradable asset $1 \tilde{X}_{1}$. Type 2 hedger get a private signal $\tilde{\theta}_{2}$ and their position on non-tradable asset $2 \tilde{X}_{2}$. And they make their decisions on how much asset $m$ they want to buy according to the private information on $\tilde{\theta}_{i}, \tilde{X}_{i}$ and information derived from equilibrium price $p$.

\subsubsection{Date 2}

The payoff of non-tradable asset 1 , asset 2, and tradable asset $m$ is realized.

\section{LEARNING FROM MARKET AND EQUILIBRIUM}

We first analyze the optimal problem of different type of hedgers. Through utility maximization conditional on the information they get, we can get hedgers' demand function. They make their demand decision after observing private signal and their non-tradable asset position. They also get some information about opponents' private information through equilibrium price. We use rational expectation equilibrium (REE) as equilibrium, as in Grossman and Stiglitz (1980). In the equilibrium, every hedger has maximized their expected utility based on their information set, where type 1 hedgers know $\mathcal{F}_{1}=$ $\left\{\tilde{\theta}_{1}, \tilde{X}_{1}, \tilde{p}\right\}$, while type 2 hedgers $\operatorname{know} \mathcal{F}_{2}=\left\{\tilde{\theta}_{2}, \tilde{X}_{2}, \tilde{p}\right\}$. The price is set to clear the market. Now we derive the equilibrium in detail.

\subsection{Price function}

Type 1 and type 2 hedgers trade depending on the information set $\mathcal{F}_{1}$ and $\mathcal{F}_{2}$. Hence, the equilibrium price of asset $m$ is a function of $\left(\tilde{\theta}_{1}, \tilde{\theta}_{2}, \tilde{X}_{1}, \tilde{X}_{2}\right): \tilde{p}=$ $p\left(\tilde{\theta}_{1}, \tilde{\theta}_{2}, \tilde{X}_{1}, \tilde{X}_{2}\right)$.

As in the case in most of the literature, we analyze linear equilibrium, that is the price $\tilde{p}$ is a linear function of $\left(\tilde{\theta}_{1}, \tilde{\theta}_{2}, \tilde{X}_{1}, \tilde{X}_{2}\right)$ :

$$
\tilde{p}=v+\alpha \tilde{\theta}_{1}+\beta \tilde{\theta}_{2}+\xi \tilde{X}_{1}+\eta \tilde{X}_{2}
$$

where the coefficients are determined endogenously.

We start to examine the decision of hedgers which in turn determine the information determined in the price $\tilde{p}$. And then we solve the market clearing equilibrium to find the equilibrium price and the coefficients in price function. 


\subsection{Hedgers' demand}

Type $i$ hedgers have information set $\mathcal{F}_{i}=\left\{\tilde{\theta}_{i}, \tilde{X}_{i}, \tilde{p}\right\}$. Let $E_{i}\left[\cdot \mid \mathcal{F}_{i}\right]$ denote the conditional expectation operator on their information set. They decide how to allocate their wealth in asset mand risk-free asset to maximize their expected utility

$$
E_{i}\left[-e^{-\gamma W_{i}^{2}} \mid \mathcal{F}_{i}\right]
$$

where the wealth of date $2 W_{i}^{2}$ is given by (3). Same type of hedgers will choose same risky asset mand risk-free asset in their investment because they have same information set. Due to the property of CARA utility function, the initial wealth is irrelevant to allocation decision. Thus, we transfer maximal problem above into

\section{Speculation demand Hedge demand}

$$
\max d_{i}\left[E_{i}\left(\tilde{v} \mid \mathcal{F}_{i}\right)-\tilde{p}\right]-\frac{\gamma}{2}\left[d_{i}^{2} \operatorname{Var}_{i}\left(\tilde{v} \mid \mathcal{F}_{i}\right)+2 d_{i} \tilde{X}_{i} \operatorname{Cov}_{i}\left(\tilde{v}, \tilde{u} \mid \mathcal{F}_{i}\right)\right]
$$

which equals to

$$
d_{i}\left[\bar{v}+\tilde{\theta}_{i}+E_{i}\left(\tilde{\theta}_{-i} \mid \mathcal{F}_{i}\right)-\tilde{p}\right]-\frac{\gamma}{2}\left[d_{i}^{2}\left(\operatorname{Var}_{i}\left(\tilde{\theta}_{-i} \mid \mathcal{F}_{i}\right)+\sigma_{\varepsilon 1}^{2}+\sigma_{\varepsilon 2}^{2}\right)+2 d_{i} \tilde{X}_{i} \sigma_{\varepsilon i} \sigma_{u i} \rho_{i}\right] .
$$

The first order condition is

$$
\bar{v}+\tilde{\theta}_{i}+E_{i}\left(\tilde{\theta}_{-i} \mid \mathcal{F}_{i}\right)-\tilde{p}-\gamma\left[d_{i}\left(\operatorname{Var}_{i}\left(\tilde{\theta}_{-i} \mid \mathcal{F}_{i}\right)+\sigma_{\varepsilon 1}^{2}+\sigma_{\varepsilon 2}^{2}\right)+\tilde{X}_{i} \sigma_{\varepsilon i} \sigma_{u i} \rho_{i}\right]=0 .
$$

where $-i=2$ if $i=1,-i=1$ if $i=2$.

Through F.O.C, we get the demand function of hedgers typed $i$ is

$$
\begin{aligned}
& d_{i}=\frac{\bar{v}+\tilde{\theta}_{i}+E_{i}\left(\tilde{\theta}_{-i} \mid \mathcal{F}_{i}\right)-\tilde{p}-\gamma \tilde{X}_{i} \sigma_{\varepsilon i} \sigma_{u i} \rho_{i}}{\gamma\left[\operatorname{Var}_{i}\left(\tilde{\theta}_{-i} \mid \mathcal{F}_{i}\right)+\sigma_{\varepsilon 1}^{2}+\sigma_{\varepsilon 2}^{2}\right]} \\
& =\frac{\bar{v}+\tilde{\theta}_{i}+E_{i}\left(\tilde{\theta}_{-i} \mid \mathcal{F}_{i}\right)-\tilde{p}}{\gamma\left[\operatorname{Var}_{i}\left(\tilde{\theta}_{-i} \mid \mathcal{F}_{i}\right)+\sigma_{\varepsilon 1}^{2}+\sigma_{\varepsilon 2}^{2}\right]}-\frac{\tilde{X}_{i} \sigma_{\varepsilon i} \sigma_{u i} \rho_{i}}{\operatorname{Var}_{i}\left(\tilde{\theta}_{-i} \mid \mathcal{F}_{i}\right)+\sigma_{\varepsilon 1}^{2}+\sigma_{\varepsilon 2}^{2}} . \\
& \text { L }
\end{aligned}
$$

Observe that the demand is constituted by two parts. The first part originates from gaining benefit through the payoff taken by asset $m$, we call it speculation demand. And the second part originates from deducing risk exposure of non-tradable asset $i$. Speculate demand depends on the private signal hedgers get, it is always positive because of risk aversion and uncertainty compensation. Hedge demand depends on the position of hedgers on non-tradable asset and correlation ofpayoff between asset $m$ and asset $i$. The signal of hedge demand is determined by position of risk exposure. When $\tilde{X}_{i}>0$ and $\rho_{i}>0$, there is a long position on the asset $i$ and the payoff of asset $i$ is positively related to asset $m$, then hedgers will buy less asset $m$ to reduce their risk. When $\tilde{X}_{i}>0$ and $\rho_{i}<0$, there is a long position on the asset $i$ and the payoff of asset $i$ is negatively related to asset $m$, then hedgers will buy more asset $m$ to reduce their risk. When $\tilde{X}_{i}<0$ and $\rho_{i}>0$, there is a short position on the asset $i$ and the payoff of asset $i$ is positively related to asset $m$, then hedgers will buy more asset $m$ to reduce their risk. When $\tilde{X}_{i}<0$ and $\rho_{i}<0$, there is a short position on the asset $i$ and the payoff of asset $i$ is negatively related to asset $m$, then hedgers will buy less asset $m$ to reduce their risk. And hedge demand is also influenced by volatility of the payoff of asset $i$ and $\varepsilon_{i}$.

According to market clearing condition, we have

$$
\mu \frac{\bar{v}+\tilde{\theta}_{1}+E_{1}\left(\tilde{\theta}_{2} \mid \mathcal{F}_{1}\right)-\tilde{p}-\gamma \tilde{X}_{1} \sigma_{\varepsilon i} \sigma_{u i} \rho_{1}}{\gamma\left[\operatorname{Var}_{1}\left(\tilde{\theta}_{2} \mid \mathcal{F}_{1}\right)+\sigma_{\varepsilon 1}^{2}+\sigma_{\varepsilon 2}^{2}\right]}
$$




$$
+(1-\mu) \frac{\bar{v}+\tilde{\theta}_{2}+E_{2}\left(\tilde{\theta}_{1} \mid \mathcal{F}_{2}\right)-\tilde{p}-\gamma \tilde{X}_{2} \sigma_{\varepsilon i} \sigma_{u i} \rho_{2}}{\gamma\left[\operatorname{Var}_{2}\left(\tilde{\theta}_{1} \mid \mathcal{F}_{2}\right)+\sigma_{\varepsilon 1}^{2}+\sigma_{\varepsilon 2}^{2}\right]}=n
$$

For type 1 hedgers, we have

$$
\begin{aligned}
\tilde{\theta}_{2}-\gamma \sigma_{\varepsilon 2} \sigma_{u 2} \rho_{2} \tilde{X}_{2}=\frac{\gamma\left[\operatorname{Var}_{2}\left(\tilde{\theta}_{1} \mid \mathcal{F}_{2}\right)+\sigma_{\varepsilon 1}^{2}+\sigma_{\varepsilon 2}^{2}\right]}{1-\mu} \\
{\left[n-\mu \frac{\bar{v}+\tilde{\theta}_{1}+E_{1}\left(\tilde{\theta}_{2} \mid \mathcal{F}_{1}\right)-\tilde{p}-\gamma \tilde{X}_{1} \sigma_{\varepsilon 1} \sigma_{u 1} \rho_{1}}{\gamma\left[\operatorname{Var}_{1}\left(\tilde{\theta}_{2} \mid \mathcal{F}_{2}\right)+\sigma_{\varepsilon 1}^{2}+\sigma_{\varepsilon 2}^{2}\right]}-(1-\mu) \frac{\bar{v}+E_{2}\left(\tilde{\theta}_{1} \mid \mathcal{F}_{2}\right)-\tilde{p}}{\gamma\left[\operatorname{Var}_{2}\left(\tilde{\theta}_{1} \mid \mathcal{F}_{2}\right)+\sigma_{\varepsilon 1}^{2}+\sigma_{\varepsilon 2}^{2}\right]}\right] . }
\end{aligned}
$$

Let $\tilde{S}_{1} \equiv \tilde{\theta}_{2}+s_{1}^{-1} \tilde{X}_{2}$, where $s_{1}=-\frac{1}{\gamma \sigma_{\varepsilon 2} \sigma_{u 2} \rho_{2}}<0$. Thus, we have $E_{1}\left(\tilde{\theta}_{2} \mid \mathcal{F}_{1}\right)=\frac{s_{1}^{2} \tau_{X 2} \tilde{S}_{1}}{\tau_{\theta 2}+s_{1}^{2} \tau_{X 2}}$ and $\operatorname{Var}_{1}\left(\tilde{\theta}_{2} \mid \tilde{\theta}_{1}, \tilde{X}_{1}, \tilde{p}\right)=$ $\frac{1}{\tau_{\theta 2}+s_{1}^{2} \tau_{X 2}}$.

For type 2 hedgers, we have

$$
\begin{aligned}
\tilde{\theta}_{1}-\gamma \sigma_{\varepsilon 1} \sigma_{u 1} \rho_{1} \tilde{X}_{1}=\frac{\gamma\left[\operatorname{Var}_{1}\left(\tilde{\theta}_{2} \mid \tilde{p}\right)+\sigma_{\varepsilon 1}^{2}+\sigma_{\varepsilon 2}^{2}\right]}{\mu} \\
{\left[n-(1-\mu) \frac{\bar{v}+\tilde{\theta}_{2}+E_{2}\left(\tilde{\theta}_{1} \mid \tilde{p}\right)-\tilde{p}-\gamma \tilde{X}_{2} \sigma_{\varepsilon i} \sigma_{u i} \rho_{2}}{\gamma\left[\operatorname{Var}_{2}\left(\tilde{\theta}_{1} \mid \tilde{p}\right)+\sigma_{\varepsilon 1}^{2}+\sigma_{\varepsilon 2}^{2}\right]}-\mu \frac{\bar{v}+E_{1}\left(\tilde{\theta}_{2} \mid \tilde{p}\right)-\tilde{p}}{\gamma\left[\operatorname{Var}_{1}\left(\tilde{\theta}_{2} \mid \tilde{p}\right)+\sigma_{\varepsilon 1}^{2}+\sigma_{\varepsilon 2}^{2}\right]}\right] . }
\end{aligned}
$$

Let $\tilde{S}_{2} \equiv \tilde{\theta}_{1}+s_{2}^{-1} \tilde{X}_{1}$, where $s_{2}=-\frac{1}{\gamma \sigma_{\varepsilon 1} \sigma_{u 1} \rho_{1}}<0$. Thus, we have $E_{2}\left(\tilde{\theta}_{1} \mid \mathcal{F}_{2}\right)=\frac{s_{2}^{2} \tau_{X 1} \tilde{S}_{2}}{\tau_{\theta 1}+s_{2}^{2} \tau_{X 1}}$ and $\operatorname{Var}_{2}\left(\tilde{\theta}_{1} \mid \mathcal{F}_{2}\right)=\frac{1}{\tau_{\theta 1}+s_{2}^{2} \tau_{X 1}}$.

Through market clearing price, both types hedgers get some information about their counterparts' private signal and non-tradable asset position. Therefore, the demands of type 1 hedgers and type 2 hedgers are respectively

$$
\begin{array}{r}
d_{1}=\frac{\bar{v}+\tilde{\theta}_{1}+\frac{s_{1}^{2} \tau_{X 2} \tilde{s}_{1}}{\tau_{\theta 2}+s_{1}^{2} \tau_{X 2}}-\tilde{p}-\gamma \tilde{X}_{1} \sigma_{\varepsilon 1} \sigma_{u 1} \rho_{1}}{\gamma\left[\frac{1}{\tau_{\theta 2}+s_{1}^{2} \tau_{X 2}}+\sigma_{\varepsilon 1}^{2}+\sigma_{\varepsilon 2}^{2}\right]}, \\
d_{2}=\frac{\bar{v}+\tilde{\theta}_{2}+\frac{s_{2}^{2} \tau_{X 1} \tilde{s}_{2}}{\tau_{\theta 1}+s_{2}^{2} \tau_{X 1}}-\tilde{p}-\gamma \tilde{X}_{i} \sigma_{\varepsilon i} \sigma_{u i} \rho_{i}}{\gamma\left[\frac{1}{\tau_{\theta 1}+s_{2}^{2} \tau_{X 1}}+\sigma_{\varepsilon 1}^{2}+\sigma_{\varepsilon 2}^{2}\right]} .
\end{array}
$$

\subsection{Equilibrium}

Definition 1. Given any hedgers constitution proportion $\mu$ and tradable risky asset supply $n$, an equilibrium $\left(d_{1}\left(\tilde{\theta}_{1}, \tilde{X}_{1}, \tilde{p}\right), d_{2}\left(\tilde{\theta}_{2}, \tilde{X}_{2}, \tilde{p}\right), \tilde{p}\right)$ is such that

1. Given any $\tilde{\theta}_{i}, \tilde{X}_{i}, \tilde{p}, d_{i}$ maximize (4).

2. $\tilde{p}$ makes market clear, that is

$$
\mu d_{1}\left(\tilde{\theta}_{1}, \tilde{X}_{1}, \tilde{p}\right)+(1-\mu) d_{2}\left(\tilde{\theta}_{2}, \tilde{X}_{2}, \tilde{p}\right)=n .
$$

We have mentioned that the sum of demands of hedgers has to be equal to the supply of asset $m$. Substitute demands (11), (12)into (13), we get asset $m$ market clearing condition

$$
\begin{aligned}
& \mu \frac{\bar{v}+\tilde{\theta}_{1}+\frac{s_{1}^{2} \tau_{X 2} \tilde{s}_{1}}{\tau_{\theta 2}+s_{1}^{2} \tau_{X 2}}-\tilde{p}-\gamma \tilde{X}_{1} \sigma_{\varepsilon 1} \sigma_{u 1} \rho_{1}}{\gamma\left[\frac{1}{\tau_{\theta 2}+s_{1}^{2} \tau_{X 2}}+\sigma_{\varepsilon 1}^{2}+\sigma_{\varepsilon 2}^{2}\right]} \\
& \quad+(1-\mu) \frac{\bar{v}+\tilde{\theta}_{2}+\frac{s_{2}^{2} \tau_{X 1} \tilde{s}_{2}}{\tau_{\theta 1}+s_{2}^{2} \tau_{X 1}}-\tilde{p}-\gamma \tilde{X}_{i} \sigma_{\varepsilon i} \sigma_{u i} \rho_{i}}{\gamma\left[\frac{1}{\tau_{\theta 1}+s_{2}^{2} \tau_{X 1}}+\sigma_{\varepsilon 1}^{2}+\sigma_{\varepsilon 2}^{2}\right]}=n .
\end{aligned}
$$

Solve this equation, we have followed proposition 1.

Proposition 1. For any given $\mu>0$, there exists a unique linear rational expected equilibrium

$$
\tilde{p}=\bar{v}-\frac{n}{\Delta}+\frac{\alpha_{1}+\alpha_{2}}{\Delta} \tilde{\theta}_{1}+\frac{\beta_{1}+\beta_{2}}{\Delta} \tilde{\theta}_{2}+\frac{\xi_{1}+\xi_{2}}{\Delta} \tilde{X}_{1}+\frac{\eta_{1}+\eta_{2}}{\Delta} \tilde{X}_{2}
$$


where

$$
\begin{aligned}
& \Delta=\frac{\Delta}{\left(\tau_{\theta 2}+s_{1}^{2} \tau_{X 2}\right)^{-1}+\sigma_{\varepsilon 1}^{2}+\sigma_{\varepsilon 2}^{2}}+\frac{\mu}{\left(\tau_{\theta 1}+s_{2}^{2} \tau_{X 1}\right)^{-1}+\sigma_{\varepsilon 1}^{2}+\sigma_{\varepsilon 2}^{2},}, \\
& \alpha_{1}=\frac{\mu}{\left(\tau_{\theta 2}+s_{1}^{2} \tau_{X 2}\right)^{-1}+\sigma_{\varepsilon 1}^{2}+\sigma_{\varepsilon 2}^{2}}, \\
& \alpha_{2}=\frac{(1-\mu) s_{2}^{2} \tau_{X 1}}{\left(\tau_{\theta 1}+s_{2}^{2} \tau_{X 1}\right)\left[\left(\tau_{\theta 1}+s_{2}^{2} \tau_{X 1}\right)^{-1}+\sigma_{\varepsilon 1}^{2}+\sigma_{\varepsilon 2}^{2}\right]}, \\
& \beta_{1}=\frac{\mu s_{1}^{2} \tau_{X 2}}{\left(\tau_{\theta 2}+s_{1}^{2} \tau_{X 2}\right)\left[\left(\tau_{\theta 2}+s_{1}^{2} \tau_{X 2}\right)^{-1}+\sigma_{\varepsilon 1}^{2}+\sigma_{\varepsilon 2}^{2}\right]^{\prime}}, \\
& \xi_{2}=\frac{\beta_{2}=\frac{1-\mu}{\left(\tau_{\theta 1}+s_{2}^{2} \tau_{X 1}\right)^{-1}+\sigma_{\varepsilon 1}^{2}+\sigma_{\varepsilon 2}^{2}},}{\left.\left(\tau_{\theta 1}+s_{2}^{2} \tau_{X 1}\right)\left[\left(\tau_{\theta 1}+s_{2}^{2} \tau_{X 1}\right)^{-1}+\sigma_{\varepsilon 1}^{2}+\sigma_{\varepsilon 2}^{2}\right]^{2} \tau_{X 2}\right)^{-1}+\sigma_{\varepsilon 1}^{2}+\sigma_{\varepsilon 2}^{2}}, \\
& \eta_{1}=\frac{(1-\mu) s_{2} \tau_{X 1}}{\left(\tau_{\theta 2}+s_{1}^{2} \tau_{X 2}\right)\left[\left(\tau_{\theta 2}+s_{1}^{2} \tau_{X 2}\right)^{-1}+\sigma_{\varepsilon 1}^{2}+\sigma_{\varepsilon 2}^{2}\right]^{\prime}} \\
& \eta_{2}=-\frac{(1-\mu) \gamma \sigma_{\varepsilon 2} \sigma_{u 2} \rho_{2}}{\left(\tau_{\theta 1}+s_{2}^{2} \tau_{X 1}\right)^{-1}+\sigma_{\varepsilon 1}^{2}+\sigma_{\varepsilon 2}^{2}} .
\end{aligned}
$$

\section{PRICE DETERMINATION}

The price is determined by demands of different types of hedgers, it depends on the hedgers' private information about private signal $\tilde{\theta}_{i}$ and non-tradable asset position $\tilde{X}_{i}$. Price also transfer information about counterpart' private information through market equilibrium. We study what factors influence the price and the sensitivity of price, how they act, and their correlation with information transfer effect of price.

Firstly, we analyze the coefficient of $\tilde{\theta}_{1}$, that is $\frac{\alpha_{1}+\alpha_{2}}{\Delta}$. We can see

$$
\begin{aligned}
& \frac{\alpha_{1}}{\Delta}=\frac{\frac{\mu}{\left(\tau_{\theta 2}+s_{1}^{2} \tau_{X 2}\right)^{-1}+\sigma_{\varepsilon 1}^{2}+\sigma_{\varepsilon 2}^{2}}}{\frac{\mu}{\left(\tau_{\theta 2}+s_{1}^{2} \tau_{X 2}\right)^{-1}+\sigma_{\varepsilon 1}^{2}+\sigma_{\varepsilon 2}^{2}}+\frac{1-\mu}{\left(\tau_{\theta 1}+s_{2}^{2} \tau_{X 1}\right)^{-1}+\sigma_{\varepsilon 1}^{2}+\sigma_{\varepsilon 2}^{2}}} \\
& \frac{\alpha_{2}}{\Delta}=\frac{\frac{(1-\mu) s_{2}^{2} \tau_{X 1}}{\left(\tau_{\theta 1}+s_{2}^{2} \tau_{X 1}\right)\left[\left(\tau_{\theta 1}+s_{2}^{2} \tau_{X 1}\right)^{-1}+\sigma_{\varepsilon 1}^{2}+\sigma_{\varepsilon 2}^{2}\right]}}{\frac{\mu}{\left(\tau_{\theta 2}+s_{1}^{2} \tau_{X 2}\right)^{-1}+\sigma_{\varepsilon 1}^{2}+\sigma_{\varepsilon 2}^{2}}+\frac{1-\mu}{\left(\tau_{\theta 1}+s_{2}^{2} \tau_{X 1}\right)^{-1}+\sigma_{\varepsilon 1}^{2}+\sigma_{\varepsilon 2}^{2}}} \\
&<\frac{\frac{1-\mu}{\left(\tau_{\theta 1}+s_{2}^{2} \tau_{X 1}\right)^{-1}+\sigma_{\varepsilon 1}^{2}+\sigma_{\varepsilon 2}^{2}}}{\frac{\mu}{\left(\tau_{\theta 2}+s_{1}^{2} \tau_{X 2}\right)^{-1}+\sigma_{\varepsilon 1}^{2}+\sigma_{\varepsilon 2}^{2}}+\frac{1-\mu}{\left(\tau_{\theta 1}+s_{2}^{2} \tau_{X 1}\right)^{-1}+\sigma_{\varepsilon 1}^{2}+\sigma_{\varepsilon 2}^{2}}} .
\end{aligned}
$$

Sum (15)and (16), we have

$$
\frac{\partial \tilde{p}}{\partial \tilde{\theta}_{1}}=\frac{\alpha_{1}}{\Delta}+\frac{\alpha_{2}}{\Delta}<1=\frac{\partial \tilde{v}}{\partial \tilde{\theta}_{1}}
$$

$\frac{\alpha_{1}}{\Delta}$ is type 1 hedgers' influence on price, type 1 hedgers have full knowledge of $\tilde{\theta}_{1}$, thus the change of $\tilde{\theta}_{1}$ can be completely responded in type 1 hedgers' demand function. $\frac{\alpha_{2}}{\Delta}$ is type 2 hedgers' influence on price, type 2 hedgers do not fully know $\tilde{\theta}_{1}$, they can only speculate some incomplete information about $\tilde{\theta}_{1}$ through price. Thus, their demands do not 
fully respond to the change of $\tilde{\theta}_{1}$. Finally, even in competitive market, the price can not fully respond to change of payoff because of asymmetric information. The difference between asymmetric information and complete information relies on

$$
T_{1} \equiv \frac{s_{2}^{2} \tau_{X 1}}{\tau_{\theta 1}+s_{2}^{2} \tau_{X 1}}=\frac{\tau_{X 1}}{\tau_{\theta 1}\left(\gamma \sigma_{\varepsilon 1} \sigma_{u 1} \rho_{1}\right)^{2}+\tau_{X 1}}
$$

Higher $T_{1}$, the market is more complete. As $T_{1}$ increases, $\frac{\alpha_{2}}{\Delta}$ gradually approaches to

$$
\frac{\frac{1-\mu}{\left(\tau_{\theta 1}+s_{2}^{2} \tau_{X 1}\right)^{-1}+\sigma_{\varepsilon 1}^{2}+\sigma_{\varepsilon 2}^{2}}}{\frac{\mu}{\left(\tau_{\theta 2}+s_{1}^{2} \tau_{X 2}\right)^{-1}+\sigma_{\varepsilon 1}^{2}+\sigma_{\varepsilon 2}^{2}}+\frac{1-\mu}{\left(\tau_{\theta 1}+s_{2}^{2} \tau_{X 1}\right)^{-1}+\sigma_{\varepsilon 1}^{2}+\sigma_{\varepsilon 2}^{2}}} .
$$

To study what influence the responding of price to $\tilde{\theta}_{1}$, we try to find the factors influencing $T_{1}$. At first, $\frac{\partial T_{1}}{\partial \tau_{X 1}}>0$ means price responds more sensitively to $\tilde{\theta}_{1}$ with the precision of $\tilde{X}_{1}$ increasing. It is because as the precision of $\tilde{X}_{1}$ increases itbecomes more stable, which means through price, type 2 hedgers can estimate $\tilde{\theta}_{1}$ more accurately. Thus, type 2 hedgers have more information about $\tilde{\theta}_{1}$ which makes price more efficient. Especially, $\tau_{X 1}=\infty$, that implies $\tilde{X}_{1}$ becomes constant. Type 2 hedger can perfectly speculate the value of $\tilde{\theta}_{1}$ through equilibrium price $\lim _{\tau_{X 1} \rightarrow \infty} T_{1}=1$. Next, we have $\frac{\partial T_{1}}{\partial \tau_{\theta 1}}<0$ implying that as precision of prior belief on $\tilde{\theta}_{1}$ decreases, the price responds more sensitively to $\tilde{\theta}_{1}$. As $\tau_{\theta 1}$ decreases, type 2 hedgers rely less on prior belief on $\tilde{\theta}_{1}$ and their speculation about $\tilde{\theta}_{1}$ will rely more on the price signal they get from trading. In reverse, price can fully reflect the change of $\tilde{\theta}_{1}$. When there is no prior belief on $\tilde{\theta}_{1}$, type 2 hedgers will fully trust the signal they get from price, and price will fully respond to the change of $\tilde{\theta}_{1}, \lim _{\tau_{\theta 1} \rightarrow 0} T_{1}=1$. Then we have $\frac{\partial T_{1}}{\partial\left|\rho_{1}\right|}<0$. As the absolute value of correlation between asset $m$ and asset 1 decreases, the price is more sensitive to the change of $\tilde{\theta}_{1}$. That is because type 1 hedgers' demand is less sensitive to $\tilde{X}_{1}$ which makes type 2 hedgers' speculation about $\tilde{\theta}_{1}$ more accurate. The asymmetric information problem weakens, price responds more sensitively to $\tilde{\theta}_{1}, \lim _{\left|\rho_{1}\right| \rightarrow 0} T_{1}=1$.

As for the coefficient of $\tilde{\theta}_{2}$, that is $\frac{\beta_{1}+\beta_{2}}{\Delta}$. We can see

$$
\begin{aligned}
& \frac{\beta_{1}}{\Delta}=\frac{\frac{\mu s_{1}^{2} \tau_{X 2}}{\left(\tau_{\theta 2}+s_{1}^{2} \tau_{X 2}\right)\left[\left(\tau_{\theta 2}+s_{1}^{2} \tau_{X 2}\right)^{-1}+\sigma_{\varepsilon 1}^{2}+\sigma_{\varepsilon 2}^{2}\right]}}{\frac{\mu}{\left(\tau_{\theta 2}+s_{1}^{2} \tau_{X 2}\right)^{-1}+\sigma_{\varepsilon 1}^{2}+\sigma_{\varepsilon 2}^{2}}+\frac{1-\mu}{\left(\tau_{\theta 1}+s_{2}^{2} \tau_{X 1}\right)^{-1}+\sigma_{\varepsilon 1}^{2}+\sigma_{\varepsilon 2}^{2}}} \\
& <\frac{\frac{\mu}{\left(\tau_{\theta 2}+s_{1}^{2} \tau_{X 2}\right)^{-1}+\sigma_{\varepsilon 1}^{2}+\sigma_{\varepsilon 2}^{2}}}{\frac{\mu}{\left(\tau_{\theta 2}+s_{1}^{2} \tau_{X 2}\right)^{-1}+\sigma_{\varepsilon 1}^{2}+\sigma_{\varepsilon 2}^{2}}+\frac{1-\mu}{\left(\tau_{\theta 1}+s_{2}^{2} \tau_{X 1}\right)^{-1}+\sigma_{\varepsilon 1}^{2}+\sigma_{\varepsilon 2}^{2}}} \\
& \frac{\beta_{2}}{\Delta}=\frac{\frac{1-\mu}{\left(\tau_{\theta 1}+s_{2}^{2} \tau_{X 1}\right)^{-1}+\sigma_{\varepsilon 1}^{2}+\sigma_{\varepsilon 2}^{2}}}{\frac{\mu}{\left(\tau_{\theta 2}+s_{1}^{2} \tau_{X 2}\right)^{-1}+\sigma_{\varepsilon 1}^{2}+\sigma_{\varepsilon 2}^{2}}+\frac{1-\mu}{\left(\tau_{\theta 1}+s_{2}^{2} \tau_{X 1}\right)^{-1}+\sigma_{\varepsilon 1}^{2}+\sigma_{\varepsilon 2}^{2}}}
\end{aligned}
$$

Sum (15)and (16), we have

$$
\frac{\partial \tilde{p}}{\partial \tilde{\theta}_{2}}=\frac{\beta_{1}}{\Delta}+\frac{\beta_{2}}{\Delta}<1=\frac{\partial \tilde{v}}{\partial \tilde{\theta}_{2}}
$$

$\frac{\beta_{1}}{\Delta}$ is type 1 hedgers' influence on price, type 1 hedgers do not fully know $\tilde{\theta}_{2}$, they can only speculate some incomplete information about $\tilde{\theta}_{2}$ through price. Thus, their demands do not fully respond to the change of $\tilde{\theta}_{2} \cdot \frac{\beta_{2}}{\Delta}$ is type 2 hedgers' influence on price, type 2 hedgers have full knowledge of $\tilde{\theta}_{2}$, thus the change of $\tilde{\theta}_{2}$ can be completely responded in type 2 
hedgers' demand function. Finally, even in competitive market, the price cannot fully respond to change of payoff because of asymmetric information.

Similarly, we can define $T_{2}=\frac{s_{1}^{2} \tau_{X 2}}{\tau_{\theta 2}+s_{1}^{2} \tau_{X 2}}=\frac{\tau_{X 2}}{\tau_{\theta 2}\left(\gamma \sigma_{\varepsilon 2} \sigma_{u 2} \rho_{2}\right)^{2}+\tau_{X 2}}$ and the analysis on the influence of $\tau_{X 2}, \tau_{\theta 2}, \rho_{2}$ is similar. Secondly, we analyze the coefficient of $\tilde{X}_{1}$ :

$$
\begin{gathered}
\frac{\xi_{1}}{\Delta}=\frac{-\frac{\mu \gamma \sigma_{\varepsilon 1} \sigma_{u 1} \rho_{1}}{\frac{\mu}{\left(\tau_{\theta 2}+s_{1}^{2} \tau_{X 2}\right)^{-1}+\sigma_{\varepsilon 1}^{2}+\sigma_{\varepsilon 2}^{2}}}}{\frac{\mu}{\left(\tau_{\theta 2}+s_{1}^{2} \tau_{X 2}\right)^{-1}+\sigma_{\varepsilon 1}^{2}+\sigma_{\varepsilon 2}^{2}}+\frac{1-\mu}{\left(\tau_{\theta 1}+s_{2}^{2} \tau_{X 1}\right)^{-1}+\sigma_{\varepsilon 1}^{2}+\sigma_{\varepsilon 2}^{2}}}, \\
\frac{\xi_{2}}{\Delta}=\frac{\frac{(1-\mu) s_{2} \tau_{X 1}}{\left(\tau_{\theta 1}+s_{2}^{2} \tau_{X 1}\right)\left[\left(\tau_{\theta 1}+s_{2}^{2} \tau_{X 1}\right)^{-1}+\sigma_{\varepsilon 1}^{2}+\sigma_{\varepsilon 2}^{2}\right]}}{\frac{\mu}{\left(\tau_{\theta 2}+s_{1}^{2} \tau_{X 2}\right)^{-1}+\sigma_{\varepsilon 1}^{2}+\sigma_{\varepsilon 2}^{2}}+\frac{1-\mu}{\left(\tau_{\theta 1}+s_{2}^{2} \tau_{X 1}\right)^{-1}+\sigma_{\varepsilon 1}^{2}+\sigma_{\varepsilon 2}^{2}}}
\end{gathered}
$$

where $\frac{\xi_{1}}{\Delta}$ is type 1 hedgers' influence on price, $\frac{\xi_{2}}{\Delta}$ is type 2 hedgers' influence on price. If correlation of payoff between asset $m$ and asset 1 is negative, that is $\rho_{1}<0$, as $\tilde{X}_{1}$ increases, type 1 hedgers need more asset $m$ to hedge the risk exposure from asset 1 . Thus, the hedge demand increases, which increases the price. And this sensitivity can be enforced by lower $\rho_{1}$. As $\sigma_{u 1}$ increases, hedge demand become more sensitive to $\tilde{X}_{1}$, which makes price more sensitive to $\tilde{X}_{1}$. And when $\rho_{1}<0, \frac{\xi_{2}}{\Delta}>$ 0 , which implies as $\tilde{X}_{1}$ increases type 2 hedgers will have positive influence on price. It can be explained that increasing of $\tilde{X}_{1}$ also increases the signal $\tilde{S}_{2}$ that type 2 hedgers get from the market. This increases the type 2 hedgers' estimation of $\tilde{\theta}_{1}$, increases the speculation demand by type 2 hedgers, and increases market price.If correlation of payoff between asset $m$ and asset 1 is positive, that is $\rho_{1}>0$, as $\tilde{X}_{1}$ increases, type 1 hedgers need less asset $m$ to avoid taking too much the risk exposure from asset 1 . Thus, the hedge demand decreases, which decreases the price. And this sensitivity can be enforced by higher $\rho_{1}$. As $\sigma_{u 1}$ increases, hedge demand become more sensitive to $\tilde{X}_{1}$, which makes price more sensitive to $\tilde{X}_{1}$. And when $\rho_{1}>0, \frac{\xi_{2}}{\Delta}<0$, which implies as $\tilde{X}_{1}$ increases type 2 hedgers will have negative influence on price. It can be explained that increasing of $\tilde{X}_{1}$ decreases the signal $\tilde{S}_{2}$ that type 2 hedgers get from the market. This decrease the type 2 hedgers' estimation of $\tilde{\theta}_{1}$, decreases the speculation demand by type 2 hedgers, and decreases market price. The effects of both type hedgers are consistent no matter what sign $\rho_{1}$ has.

As for the coefficient of $\tilde{X}_{2}$, that is $\frac{\eta_{1}+\eta_{2}}{\Delta}$,

$$
\begin{aligned}
& \frac{\eta_{1}}{\Delta}=\frac{\frac{\mu s_{1} \tau_{X 2}}{\left(\tau_{\theta 2}+s_{1}^{2} \tau_{X 2}\right)\left[\left(\tau_{\theta 2}+s_{1}^{2} \tau_{X 2}\right)^{-1}+\sigma_{\varepsilon 1}^{2}+\sigma_{\varepsilon 2}^{2}\right]}}{\frac{\mu}{\left(\tau_{\theta 2}+s_{1}^{2} \tau_{X 2}\right)^{-1}+\sigma_{\varepsilon 1}^{2}+\sigma_{\varepsilon 2}^{2}}+\frac{1-\mu}{\left(\tau_{\theta 1}+s_{2}^{2} \tau_{X 1}\right)^{-1}+\sigma_{\varepsilon 1}^{2}+\sigma_{\varepsilon 2}^{2}}}, \\
& \frac{\eta_{2}}{\Delta}=\frac{-\frac{(1-\mu) \gamma \sigma_{\varepsilon 2} \sigma_{u 2} \rho_{2}}{\left(\tau_{\theta 1}+s_{2}^{2} \tau_{X 1}\right)^{-1}+\sigma_{\varepsilon 1}^{2}+\sigma_{\varepsilon 2}^{2}}}{\frac{1-\mu}{\left(\tau_{\theta 2}+s_{1}^{2} \tau_{X 2}\right)^{-1}+\sigma_{\varepsilon 1}^{2}+\sigma_{\varepsilon 2}^{2}}+\frac{1}{\left(\tau_{\theta 1}+s_{2}^{2} \tau_{X 1}\right)^{-1}+\sigma_{\varepsilon 1}^{2}+\sigma_{\varepsilon 2}^{2}}}
\end{aligned}
$$

where $\frac{\eta_{1}}{\Delta}$ is type 1 hedgers' influence on price, $\frac{\eta_{2}}{\Delta}$ is type 2 hedgers' influence on price. If correlation of payoff between asset $m$ and asset 2 is negative, that is $\rho_{2}<0$, as $\tilde{X}_{2}$ increases, type 2 hedgers need more asset $m$ to hedge the risk exposure from asset 2 . Thus, the hedge demand of type 2 hedgers increases, which increases the price. And this sensitivity can be enforced by lower $\rho_{2}$. As $\sigma_{u 2}$ increases, hedge demand become more sensitive to $\tilde{X}_{2}$, which makes price more sensitive to $\tilde{X}_{2}$. And when $\rho_{2}<0, \frac{\eta_{1}}{\Delta}>0$, which implies as $\tilde{X}_{2}$ increases type 1 hedgers will have positive influence on price. It can be explained that increasing of $\tilde{X}_{2}$ also increases the signal $\tilde{S}_{1}$ that type 1 hedgers get from the market. This increases the type 1 hedgers' 
estimation of $\tilde{\theta}_{2}$, increases the speculation demand by type 1 hedgers, and increases market price.If correlation of payoff between asset $m$ and asset 2 is positive, that is $\rho_{2}>0$, as $\tilde{X}_{2}$ increases, type 1 hedgers need less asset $m$ to avoid taking too much the risk exposure from asset 2 . Thus, the hedge demand decreases, which decreases the price. And this sensitivity can be enforced by higher $\rho_{2}$. As $\sigma_{u 2}$ increases, hedge demand become more sensitive to $\tilde{X}_{2}$, which makes price more sensitive to $\tilde{X}_{2}$.And when $\rho_{2}>0, \frac{\eta_{1}}{\Delta}<0$, which implies as $\tilde{X}_{2}$ increases type 1 hedgers will have negative influence on price. It can be explained that increasing of $\tilde{X}_{2}$ decreases the signal $\tilde{S}_{1}$ that type 1 hedgers get from the market. This decreases the type 1 hedgers' estimation of $\tilde{\theta}_{2}$, decreases the speculation demand by type 1 hedgers, and decreases market price.The effects of both type hedgers are consistent no matter what sign $\rho_{2}$ has.

To summarize the analysis above, we have proposition 2 and proposition 3.

Proposition 2.As volatility of $\tilde{X}_{1}$ decreases, type 2 hedgers can speculate on $\tilde{\theta}_{1}$ more accurately, and increases the sensitivity of price to $\tilde{\theta}_{1}$. As volatility of prior belief on $\tilde{\theta}_{1}$ increases, type 2 hedgers rely more on price to speculate on $\tilde{\theta}_{1}$, which makes price more sensitive to $\tilde{\theta}_{1}$. As the relativity between asset 1 and asset $m$ decreases, type 2 hedger can speculate $\tilde{\theta}_{1}$ more accurately, which makes price more sensitive to $\tilde{\theta}_{1}$. As volatility of $\tilde{X}_{2}$ decreases, type 1 hedgers can speculate on $\tilde{\theta}_{2}$ more accurately, and increases the sensitivity of price to $\tilde{\theta}_{2}$. As volatility of prior belief on $\tilde{\theta}_{2}$ increases, type 1 hedgers rely more on price to speculate on $\tilde{\theta}_{2}$, which makes price more sensitive to $\tilde{\theta}_{2}$. As the relativity between asset 2 and asset $m$ decreases, type 1 hedger can speculate $\tilde{\theta}_{2}$ more accurately, which makes price more sensitive to $\tilde{\theta}_{2}$.

Proposition 3. If $\rho_{1}>0$, market price will decrease as $\tilde{X}_{1}$ increases. If $\rho_{1}<0$, market price will increase as $\tilde{X}_{1}$ increases.If $\rho_{2}>0$, market price will decrease as $\tilde{X}_{2}$ increases. If $\rho_{2}<0$, market price will increase as $\tilde{X}_{2}$ increases.

\section{PRICE INFORMATIVENESS}

In measurement of price informativeness, we try to capture the amount of uncertainty about $\tilde{\theta}_{-i}$ that is reduced by observing asset $m$ price $\tilde{p}$ based on knowingtheir non-tradable asset position $\tilde{X}_{i}$, and their private signal $\tilde{\theta}_{i}$ for $i \in\{1,2\}$. Thus, we define type 1 and type 2hedgers' price informativeness as:

$$
\begin{aligned}
& I_{1} \equiv \frac{\operatorname{Var}_{1}\left(\tilde{\theta}_{2} \mid \tilde{\theta}_{1}, \tilde{X}_{1}\right)}{\operatorname{Var}_{1}\left(\tilde{\theta}_{2} \mid \tilde{\theta}_{1}, \tilde{X}_{1}, \tilde{p}\right)}-1=s_{1}^{2} \frac{\tau_{X 2}}{\tau_{\theta 2}}=\frac{\tau_{X 2}}{\left(\gamma \sigma_{\varepsilon 2} \sigma_{u 2} \rho_{2}\right)^{2} \tau_{\theta 2}}, \\
& I_{2} \equiv \frac{\operatorname{Var}_{2}\left(\tilde{\theta}_{1} \mid \tilde{\theta}_{2}, \tilde{X}_{2}\right)}{\operatorname{Var}_{2}\left(\tilde{\theta}_{1} \mid \tilde{\theta}_{2}, \tilde{X}_{2}, \tilde{p}\right)}-1=s_{2}^{2} \frac{\tau_{X 1}}{\tau_{\theta 1}}=\frac{\tau_{X 1}}{\left(\gamma \sigma_{\varepsilon 1} \sigma_{u 1} \rho_{1}\right)^{2} \tau_{\theta 1}},
\end{aligned}
$$

where the second equality follows from $\operatorname{Var}_{i}\left(\tilde{\theta}_{-i} \mid \tilde{\theta}_{i}, \tilde{X}_{i}\right)=\operatorname{Var}\left(\tilde{\theta}_{-i}\right)=\frac{1}{\tau_{\theta-i}}$ and $\operatorname{Var}_{i}\left(\tilde{\theta}_{-i} \mid \tilde{\theta}_{i}, \tilde{X}_{i}, \tilde{p}\right)=\frac{1}{\tau_{\theta-i}+s_{i}^{2} \tau_{X-i}}$, third equality flows from $s_{i}=-\frac{1}{\gamma \sigma_{\varepsilon-i} \sigma_{u-i} \rho_{-i}}$.

Proposition 4. The price informativeness of $\tilde{\theta}_{1}$ increases with precision of $\tilde{X}_{2}, \tilde{\varepsilon}_{2}$ and decreases with precision of $\tilde{\theta}_{2}$, volatility of $\tilde{u}_{2}, \rho_{2}$ and risk aversion coefficient $\gamma$. Similarly, price informativeness of $\tilde{\theta}_{2}$ increases with precision of $\tilde{X}_{1}, \tilde{\varepsilon}_{1}$ and decreases with precision of $\tilde{\theta}_{1}$, volatility of $\tilde{u}_{1}, \rho_{1}$ and risk aversion coefficient $\gamma$.

Proof. It is easy to see from (19) and (20).

The influence of these coefficients comes from two sources. Firstly, they can influence the accuracy of estimation on counterparts' private signal and speculation demand further. Secondly, they can influence the risk exposure of holding non-tradable asset and hedging demand further. Through demand, they influence the competition of the market and price informativeness.

The decreasing of $\gamma, \sigma_{\varepsilon 1}, \sigma_{u 1}$, and $\rho_{1}$ increase the estimation accuracy of type 2 hedgers on $\tilde{\theta}_{1}$, and decrease type 1 hedgers' hedging demand. Both effects are beneficial to increase type 2 hedgers' price informativeness $I_{1}$. The increasing of $\tau_{X 1}$ increase the estimation of type 2 hedgers on $\tilde{\theta}_{1}$ which increases the price informativeness $I_{1}$. The increasing of $\tau_{\theta 1}$ decreases the price informativeness $I_{1}$, because hedgers rely more on their prior belief to decide. 
We can see the price informativeness is irrelative to the proportion of different types of hedgers. Minor type hedgers do not have information strength as other models. That is because increasing hedgers number in one type do not expose more information to the other type hedgers.

\section{COST OF CAPITAL}

We examine the implication of price informativeness on asset price and define risk premium as the cost of capital given any non-tradable asset, that is

From proposition 1, we know that

$$
C C \equiv E\left(\tilde{v}-\tilde{p} \mid \tilde{X}_{1}, \tilde{X}_{2}\right)
$$

$$
\begin{aligned}
& C C=\frac{n}{\Delta}-\frac{\xi_{1}+\xi_{2}}{\Delta} \tilde{X}_{1}-\frac{\eta_{1}+\eta_{2}}{\Delta} \tilde{X}_{2} \\
& =\frac{n}{\frac{\mu}{\left(\tau_{\theta 2}+s_{1}^{2} \tau_{X 2}\right)^{-1}+\sigma_{\varepsilon 1}^{2}+\sigma_{\varepsilon 2}^{2}}+\frac{1-\mu}{\left(\tau_{\theta 1}+s_{2}^{2} \tau_{X 1}\right)^{-1}+\sigma_{\varepsilon 1}^{2}+\sigma_{\varepsilon 2}^{2}}} \\
& +\frac{\frac{\mu \gamma \sigma_{\varepsilon 1} \sigma_{u 1} \rho_{1}}{\left(\tau_{\theta 2}+s_{1}^{2} \tau_{X 2}\right)^{-1}+\sigma_{\varepsilon 1}^{2}+\sigma_{\varepsilon 2}^{2}}-\frac{(1-\mu) s_{2} \tau_{X 1}}{\left(\tau_{\theta 1}+s_{2}^{2} \tau_{X 1}\right)\left[\left(\tau_{\theta 1}+s_{2}^{2} \tau_{X 1}\right)^{-1}+\sigma_{\varepsilon 1}^{2}+\sigma_{\varepsilon 2}^{2}\right]}}{\frac{\mu}{\left(\tau_{\theta 2}+s_{1}^{2} \tau_{X 2}\right)^{-1}+\sigma_{\varepsilon 1}^{2}+\sigma_{\varepsilon 2}^{2}}+\frac{1-\mu}{\left(\tau_{\theta 1}+s_{2}^{2} \tau_{X 1}\right)^{-1}+\sigma_{\varepsilon 1}^{2}+\sigma_{\varepsilon 2}^{2}}} \tilde{X}_{1} \\
& +\frac{\frac{(1-\mu) \gamma \sigma_{\varepsilon 2} \sigma_{u 2} \rho_{2}}{\left(\tau_{\theta 1}+s_{2}^{2} \tau_{X 1}\right)^{-1}+\sigma_{\varepsilon 1}^{2}+\sigma_{\varepsilon 2}^{2}}-\frac{\mu s_{1} \tau_{X 2}}{\left(\tau_{\theta 2}+s_{1}^{2} \tau_{X 2}\right)\left[\left(\tau_{\theta 2}+s_{1}^{2} \tau_{X 2}\right)^{-1}+\sigma_{\varepsilon 1}^{2}+\sigma_{\varepsilon 2}^{2}\right]}}{\frac{1-\mu}{\left(\tau_{\theta 2}+s_{1}^{2} \tau_{X 2}\right)^{-1}+\sigma_{\varepsilon 1}^{2}+\sigma_{\varepsilon 2}^{2}}+\frac{1-\mu}{\left(\tau_{\theta 1}+s_{2}^{2} \tau_{X 1}\right)^{-1}+\sigma_{\varepsilon 1}^{2}+\sigma_{\varepsilon 2}^{2}}} \tilde{X}_{2}
\end{aligned}
$$

If $\rho_{1}>0$, Cost of capital increases as $\tilde{X}_{1}$ increases because both type 1 hedgers' hedging demand and type 2 hedgers' speculation demand decrease which decreases the price. And risk premium increases.If $\rho_{1}<0$, Cost of capital increases as $\tilde{X}_{1}$ depends because type 1 hedgers' hedging demand increases and type 2 hedgers' speculation demand decrease. We cannot tell which effect is in dominance. The analysis on $\rho_{1}$ and $\tilde{X}_{2}$ is similar.

To further analyze the situation of $\rho_{1}<0$, we can see that the coefficient of $\tilde{X}_{1}$ is positive if $\mu=0$ and negative if $\mu=1$. By continuity, there exist a $\bar{\mu} \in(0,1)$ rendering

$$
\frac{\mu \gamma \sigma_{\varepsilon 1} \sigma_{u 1} \rho_{1}}{\left(\tau_{\theta 2}+s_{1}^{2} \tau_{X 2}\right)^{-1}+\sigma_{\varepsilon 1}^{2}+\sigma_{\varepsilon 2}^{2}}-\frac{(1-\mu) s_{2} \tau_{X 1}}{\left(\tau_{\theta 1}+s_{2}^{2} \tau_{X 1}\right)\left[\left(\tau_{\theta 1}+s_{2}^{2} \tau_{X 1}\right)^{-1}+\sigma_{\varepsilon 1}^{2}+\sigma_{\varepsilon 2}^{2}\right]}=0 .
$$

For $\mu>\bar{\mu}$, cost of capital decreases as $\tilde{X}_{1}$ increases, because increasing of hedging demands of type 1 hedgers is greater than decreasing of speculation demands of type 2 hedgers. For $\mu<\bar{\mu}$, cost of capital increases as $\tilde{X}_{1}$ increases, because increasing of hedging demands of type 1 hedgers is less than decreasing of speculation demands of type 2 hedgers. $\tilde{X}_{1}$ is similar.

\section{CONCLUSION}

In our model, we analyze the market with two types of hedgers who own different kinds of non-tradable asset and know different information about the payoff of a tradable asset. Through market clearing, they can estimate counterparts' information about the tradable asset and their position in non-tradable asset by equilibrium price. And make their decision to enter market trading.

We separate hedgers' demands into two parts: speculation demand and hedging demand. Speculation demand derives from hedger's own information about payoff of tradable asset and estimation of counterpart's information. Hedging demand derives from his position on non-tradable asset. All types of hedgers' demand decide the equilibrium price. We conclude that the market price cannot fully reflect the influence of private information because there are always some other type hedgers who do not know accurate information about the payoff. As prior belief of holding position on non-tradable becomes more inaccurate, the market price reacts less to the information because it is more difficult for different type hedgers to estimate others' private information. As prior belief of 
private information becomes more inaccurate, the price reacts more to the information because they have to rely more on price to maker estimation on others' private information. More importantly, the increasing of relativity between non-tradable asset and tradable asset decreases the accuracy of others' estimation on their private information because more hedging demand is not benefit for market efficiency but decreases it by reducing speculation demand. Further, we find that tradable asset price is positively related to non-tradable asset position if they have negative correlation. It is because non-tradable asset and tradable asset are complements if their payoff are positively related. And tradable asset price is negatively related to non-tradable asset position if they have positive correlation. It is because non-tradable asset and tradable asset are substitutes if their payoff are negatively related.

Later, we research price informativeness of market clearing. The more accurate estimation is, the more informative price is. And less hedging demands can increase price informativeness. If the correlation between non-tradable asset and tradable asset is positive, risk premium increases with non-tradable position increasing. If the correlation between non-tradable asset and tradable asset is negative, the relation between risk primum and non-tradable asset position is unclear depending on hedgers' proportion.

\section{REFERENCES}

[1] Anat R. Admati, A Noisy Rational Expectations Equilibrium for Multi-Asset Securities Markets, Econometrica, May, 1985, Vol. 53, No. 3, pp. 629-658

[2] Bruno Biais, Peter Bossaerts and Chester Spatt,Equilibrium Asset Pricing and Portfolio Choice Under Asymmetric Information,The Review of Financial Studies,Vol. 23, No. 4 (April 2010), pp. 1503-1543

[3] Bruno Biais, Peter Bossaerts and Chester Spatt, Equilibrium Asset Pricing and Portfolio Choice Under Asymmetric Information, The Review of Financial Studies, April 2010, Vol. 23, No. 4, pp. 1503-1543

[4] David Easley and Maureen O'hara, Information and the Cost of Capital, The Journal of Finance, Aug, 2004, Vol. 59, No. 4, pp. 1553-1583

[5] David Easley, Maureen O'Hara, Liyan Yang, The Review of Financial Studies, Vol. 27, No. 4, April 2014, pp. 1190-1237
[6] Hendrik Bessembinder,Systematic Risk, Hedging Pressure, and Risk Premiums in Futures Markets, The Review of Financial Studies, Vol. 5, No. 4 (1992), pp. 637-667

[7] Hong Liu, Yajun Wang, Asset Pricing Implications of Short-Sale Constraints in Imperfectly Competitive Markets, Management Science, Vol. 65, No. 9, September 2019, pp. $4422-4439$

[8] Hong Liu, Yajun Wang, Market making with asymmetric information and inventory risk, Journal of Economic Theory,Volume 163, May 2016, Pages 73-109

[9] Itay Goldstein, Yan Li and Liyan Yang, Speculation and Hedging in Segmented Markets,The Review of Financial Studies,Vol. 27, No. 3, March 2014, pp. 881-922

[10] Sanford J. Grossman and Joseph E. Stiglitz, On the Impossibility of Informationally Efficient Markets, The American Economic Review, Jun., 1980, Vol. 70, No. 3, pp. 393-408

[11] Xiaojuan Hu and Cheng-Zhong Qin, Information acquisition and welfare effect in a model of competitive financial markets,Economic Theory,September 2013, Vol. 54, No. 1, pp. $199-210$

\section{APPENDIX}

Proof of proposition 1 .

Substitute $\tilde{S}_{1} \equiv \tilde{\theta}_{2}+s_{1}^{-1} \tilde{X}_{2}$ and $\tilde{S}_{2} \equiv \tilde{\theta}_{1}+s_{2}^{-1} \tilde{X}_{1}$ into (14), we have

$\frac{1}{\gamma}\left[\frac{\mu}{\left(\tau_{\theta 2}+s_{1}^{2} \tau_{X 2}\right)^{-1}+\sigma_{\varepsilon 1}^{2}+\sigma_{\varepsilon 2}^{2}}\right.$

$\left.+\frac{1-\mu}{\left(\tau_{\theta 1}+s_{2}^{2} \tau_{X 1}\right)^{-1}+\sigma_{\varepsilon 1}^{2}+\sigma_{\varepsilon 2}^{2}}\right] \tilde{p}$

$=\frac{\bar{v}}{\gamma}\left[\frac{\mu}{\left(\tau_{\theta 2}+s_{1}^{2} \tau_{X 2}\right)^{-1}+\sigma_{\varepsilon 1}^{2}+\sigma_{\varepsilon 2}^{2}}\right.$

$\left.+\frac{1-\mu}{\left(\tau_{\theta 1}+s_{2}^{2} \tau_{X 1}\right)^{-1}+\sigma_{\varepsilon 1}^{2}+\sigma_{\varepsilon 2}^{2}}\right]-n$

$+\mu \frac{\tilde{\theta}_{1}+\frac{s_{1}^{2} \tau_{X 2}\left(\tilde{\theta}_{2}+s_{1}^{-1} \tilde{X}_{2}\right)}{\tau_{\theta 2}+s_{1}^{2} \tau_{X 2}}-\gamma \tilde{X}_{1} \sigma_{\varepsilon 1} \sigma_{u 1} \rho_{1}}{\gamma\left[\frac{1}{\tau_{\theta 2}+s_{1}^{2} \tau_{X 2}}+\sigma_{\varepsilon 1}^{2}+\sigma_{\varepsilon 2}^{2}\right]}$

$+(1-\mu) \frac{\tilde{\theta}_{2}+\frac{s_{2}^{2} \tau_{X 1}\left(\widetilde{\theta}_{1}+s_{2}^{-1} \tilde{X}_{1}\right)}{\tau_{\theta 1}+s_{2}^{2} \tau_{X 1}}-\gamma \tilde{X}_{2} \sigma_{\varepsilon 2} \sigma_{u 2} \rho_{2}}{\gamma\left[\frac{1}{\tau_{\theta 1}+s_{2}^{2} \tau_{X 1}}+\sigma_{\varepsilon 1}^{2}+\sigma_{\varepsilon 2}^{2}\right]}$

Simplify it, we get 


$$
\begin{aligned}
& \frac{1}{\gamma}\left[\frac{\mu}{\left(\tau_{\theta 2}+s_{1}^{2} \tau_{X 2}\right)^{-1}+\sigma_{\varepsilon 1}^{2}+\sigma_{\varepsilon 2}^{2}}\right. \\
& \left.+\frac{1-\mu}{\left(\tau_{\theta 1}+s_{2}^{2} \tau_{X 1}\right)^{-1}+\sigma_{\varepsilon 1}^{2}+\sigma_{\varepsilon 2}^{2}}\right] \tilde{p} \\
& =\frac{\bar{v}}{\gamma}\left[\frac{\mu}{\left(\tau_{\theta 2}+s_{1}^{2} \tau_{X 2}\right)^{-1}+\sigma_{\varepsilon 1}^{2}+\sigma_{\varepsilon 2}^{2}}\right. \\
& \left.+\frac{1-\mu}{\left(\tau_{\theta 1}+s_{2}^{2} \tau_{X 1}\right)^{-1}+\sigma_{\varepsilon 1}^{2}+\sigma_{\varepsilon 2}^{2}}\right]-n \\
& +\frac{1}{\gamma}\left[\frac{\mu}{\left(\tau_{\theta 2}+s_{1}^{2} \tau_{X 2}\right)^{-1}+\sigma_{\varepsilon 1}^{2}+\sigma_{\varepsilon 2}^{2}}\right. \\
& \left.+\frac{(1-\mu) s_{2}^{2} \tau_{X 1}}{\left(\tau_{\theta 1}+s_{2}^{2} \tau_{X 1}\right)\left[\left(\tau_{\theta 1}+s_{2}^{2} \tau_{X 1}\right)^{-1}+\sigma_{\varepsilon 1}^{2}+\sigma_{\varepsilon 2}^{2}\right]}\right] \tilde{\theta}_{1} \\
& +\frac{1}{\gamma}\left[\frac{\mu s_{1}^{2} \tau_{X 2}}{\left(\tau_{\theta 2}+s_{1}^{2} \tau_{X 2}\right)\left[\left(\tau_{\theta 2}+s_{1}^{2} \tau_{X 2}\right)^{-1}+\sigma_{\varepsilon 1}^{2}+\sigma_{\varepsilon 2}^{2}\right]}\right. \\
& \left.+\frac{1-\mu}{\left(\tau_{\theta 1}+s_{2}^{2} \tau_{X 1}\right)^{-1}+\sigma_{\varepsilon 1}^{2}+\sigma_{\varepsilon 2}^{2}}\right] \tilde{\theta}_{2} \\
& \frac{1}{\gamma}\left[-\frac{\mu \gamma \sigma_{\varepsilon 1} \sigma_{u 1} \rho_{1}}{\left(\tau_{\theta 2}+s_{1}^{2} \tau_{X 2}\right)^{-1}+\sigma_{\varepsilon 1}^{2}+\sigma_{\varepsilon 2}^{2}}\right. \\
& \left.+\frac{(1-\mu) s_{2} \tau_{X 1}}{\left(\tau_{\theta 1}+s_{2}^{2} \tau_{X 1}\right)\left[\left(\tau_{\theta 1}+s_{2}^{2} \tau_{X 1}\right)^{-1}+\sigma_{\varepsilon 1}^{2}+\sigma_{\varepsilon 2}^{2}\right]}\right] \tilde{X}_{1} \\
& \frac{1}{\gamma}\left[\frac{\mu s_{1} \tau_{X 2}}{\left(\tau_{\theta 2}+s_{1}^{2} \tau_{X 2}\right)\left[\left(\tau_{\theta 2}+s_{1}^{2} \tau_{X 2}\right)^{-1}+\sigma_{\varepsilon 1}^{2}+\sigma_{\varepsilon 2}^{2}\right]}\right. \\
& \left.-\frac{(1-\mu) \gamma \sigma_{\varepsilon 2} \sigma_{u 2} \rho_{2}}{\left(\tau_{\theta 1}+s_{2}^{2} \tau_{X 1}\right)^{-1}+\sigma_{\varepsilon 1}^{2}+\sigma_{\varepsilon 2}^{2}}\right] \tilde{X}_{2}
\end{aligned}
$$

Therefore, we have

$$
\begin{gathered}
\tilde{p}=\bar{v}-\frac{n}{\Delta}+\frac{\alpha_{1}+\alpha_{2}}{\Delta} \tilde{\theta}_{1}+\frac{\beta_{1}+\beta_{2}}{\Delta} \tilde{\theta}_{2}+\frac{\xi_{1}+\xi_{2}}{\Delta} \tilde{X}_{1} \\
+\frac{\eta_{1}+\eta_{2}}{\Delta} \tilde{X}_{2} .
\end{gathered}
$$

